\title{
High frequency conductivity of the high-mobility two-dimensional electron gas
}

\author{
P. J. Burke, I. B. Spielman, and J. P. Eisenstein \\ Condensed Matter Physics, California Institute of Technology (Caltech) Pasadena, California 91125 \\ L. N. Pfeiffer and K. W. West \\ Bell Laboratories, Lucent Technologies, Murray Hill, New Jersey 07974
}

(Received 4 June 1999; accepted for publication 10 December 1999)

\begin{abstract}
We measure the real and imaginary conductivity $\sigma(k=0, \omega)$ of a high-mobility two-dimensional electron gas (2DEG) system at frequencies below and above the momentum scattering rate. The imaginary part of the 2DEG impedance is observed to be inductive, consistent with the Drude model. Using this kinetic inductance, we construct a transmission line by capacitively coupling the 2DEG to an Al Schottky barrier gate separated by $5000 \AA$ from the 2DEG. The measured wave velocity and temperature-dependent damping of this transmission line are in good agreement with a simple Drude model. Exciting these modes is equivalent to exciting a 2D plasma mode strongly modified by the interaction between the 2DEG and the gate. (C) 2000 American Institute of Physics.
\end{abstract} [S0003-6951(00)01906-9]

One of the most fundamental properties of a twodimensional electron gas (2DEG) is the mobility of the system. The highest mobility 2DEGs are formed in GaAs/ AlGaAs heterostructures, but to date there have been no measurements of the frequency dependent conductivity $\sigma(k=0, \omega)$, hereafter referred to as $\sigma(\omega)$, in these systems at zero magnetic field. The Drude model predicts

$$
\sigma(\omega)=\frac{n e^{2} \tau_{\mathrm{tr}}}{m^{*}} \frac{1}{1+i \omega \tau_{\mathrm{tr}}},
$$

where $\tau_{\text {tr }}$ is the transport or momentum scattering time, $n$ is the electron density, and $m^{*}$ is the effective mass. The predicted impedance of a 2 DEG then contains a real and an imaginary part, which can be written as

$$
Z_{2 \mathrm{DEG}}(\omega)=R+i \omega L_{K}
$$

$L_{K}$ is referred to as the kinetic inductance, and arises from the inertia of the electrons. (Evidence for this inductive behavior in GaAs 2DEGs was reported in Ref. 1 at frequencies where $\omega \tau_{\mathrm{tr}} \sim 0.1$.) In the first half of this letter, we use vector microwave methods (where both magnitude and phase of the microwaves are measured) to measure both the real and imaginary parts of $\sigma(\omega)$ at frequencies above and below the momentum scattering rate, $\tau_{\mathrm{tr}}^{-1}$. This is possible since $\tau_{\mathrm{tr}}^{-1}$ in these high-mobility 2DEGs is in the microwave region. Optical techniques at far-infrared frequencies were used by Allen et $a .^{2}$ to measure the real part of $\sigma(\omega)$ in lower mobility silicon inversion layers. Our measurements are in good agreement with Eqs. (1) and (2) for both the real and the imaginary conductivity. In the second half of this letter, we construct and characterize a kinetic-inductance transmission line: The distributed kinetic inductance of the 2DEG and the distributed capacitance between the 2DEG and an evaporated Schottky barrier Al gate act to guide electromagnetic waves. (The optical analog of this technique was studied in Refs. 3 and 4.) This is equivalent to exciting a 2D plasmon modified by the presence of a gate.
The samples studied are GaAs/AlGaAs modulationdoped quantum wells or heterojunctions grown by molecular beam epitaxy. The samples are connected to the end of a coax in a ${ }^{3} \mathrm{He}$ immersion refrigerator. The $2 \mathrm{DEG}$ system is capacitively contacted by evaporating an Al Schottky barrier gate onto the surface of the sample; the gate to 2DEG separation $d$ is typically $5000 \AA$ (see the inset of Fig. 1.) Thus, the circuit which terminates the coax consists of two capacitive contacts in series with an ungated 2DEG.

We measure the magnitude and phase of the microwave reflection coefficient $\Gamma(\omega) \equiv V_{\text {reflected }} / V_{\text {incident }}$ off of this circuit as a function of temperature and gate voltage for several different sample geometries. From this measurement, we determine the total (complex) circuit impedance $Z(\omega)$ by inverting the standard reflection formula: $\Gamma(\omega)=[Z(\omega)$

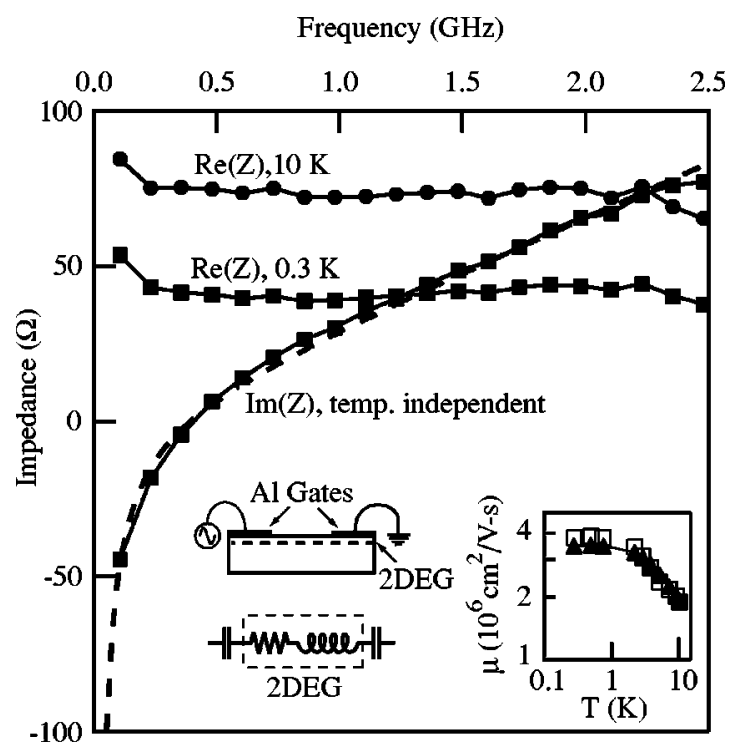

FIG. 1. Impedance vs frequency. The dashed line is a fit of the imaginary impedance to an $L C$ circuit. The squares in the inset are determined from the extrapolated dc resistance and measured density; the triangles from the frequency at which the real and imaginary impedance are equal. 


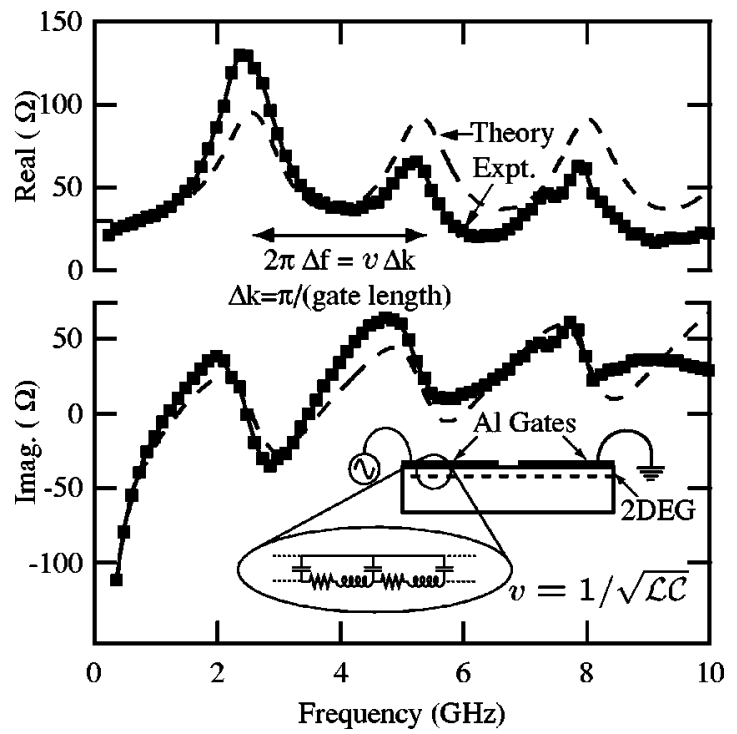

FIG. 2. Impedance vs frequency with kinetic-inductance transmission line resonances excited. $\omega \tau_{\mathrm{tr}}=1$ at $f \approx 1.25 \mathrm{GHz}$ in this sample.

$-50 \Omega] /[Z(\omega)+50 \Omega]$. At the power level used $(1 \mathrm{nW})$, the results are independent of the power indicating that Joule heating is insignificant. Corrections to $\Gamma$ due to the attenuation $(<5 \mathrm{~dB}$ up to $10 \mathrm{GHz})$ and phase delay of the coax (approx $1.5 \mathrm{~m}$ in length) are applied after the measurement.

The sample geometry is rectangular, defined either by mesa etching or direct cleaving. The mesa lengths vary between 0.5 and $3 \mathrm{~mm}$, the widths between 0.1 and $1 \mathrm{~mm}$, and the distance from the gate to the 2DEG between 1900 and $5800 \AA$. By varying the length of the ungated region, we control whether the impedance of the ungated region or of the capacitive contacts dominates the total circuit impedance. We consider the former case first.

According to Eq. (2), a capacitively contacted 2DEG will have a circuit equivalent to an $R L C$ resonator, as shown in Fig. 1. We plot in Fig. 1 the impedance versus frequency for a sample from wafer A. The 2DEG size is $510 \mu \mathrm{m}$ $\times 2560 \mu \mathrm{m}$; the lengths of the two $\mathrm{Al}$ gates are 330 and 680 $\mu \mathrm{m}$. The real impedance, $\operatorname{Re}(Z)$, is independent of frequency, in agreement with the model, and the imaginary part fits the $L C$ circuit model $\operatorname{Im}(Z)=i \omega L_{K}+1 / i \omega C$ very well. [The slight rise in $\operatorname{Re}(Z)$ at low frequencies is probably due to increased uncertainty in the measurement as $\omega \rightarrow 0$, since the capacitive impedance diverges in that limit.] We find $L_{K}=5.4 \mathrm{nH}$ and $C=24 \mathrm{pF}$, which is in good agreement with the values of 5.4 $\mathrm{nH}$ and $30 \mathrm{pF}$ calculated from the geometry and density. (The carrier density of this sample is measured in situ by measuring the capacitance versus magnetic field.)

The dc mobility of the sample can be determined in two ways from the data plotted in Fig. 1. First, the measured $\operatorname{Re}(Z)$, scaled by the geometry, gives a measure of the resistivity. This, combined with the measured density, gives the mobility. Second, the frequency at which $\operatorname{Re}(Z)=\operatorname{Im}(Z)$ is equal to $\tau_{\text {tr }}^{-1}$, which is related to the mobility $\mu$ through $\mu$ $=e \tau_{\text {tr }} / m^{*}$. [The capacitive contribution to $\operatorname{Im}(Z)$ is small at the crossover frequency in this graph.] Note that this method is independent of sample geometry. In the inset, we plot the temperature dependence of the mobility determined using both methods. The consistency between the two methods, as Downloaded 26 Feb 2006 to 131.215.240.9. Redistribution subject
TABLE I. Comparison of sample scattering rates determined by dc $\left(\tau_{\mathrm{dc}}\right)$ and microwave $\left(\tau_{\mathrm{ac}}\right)$ transport measurements.

\begin{tabular}{cccrr}
\hline \hline Wafer & $\begin{array}{c}\text { Density } \\
\left(10^{11} \mathrm{~cm}^{-2}\right)\end{array}$ & $\begin{array}{c}\text { Mobility } \\
\left(10^{6} \mathrm{~cm}^{2} / \mathrm{V} \mathrm{s}\right)\end{array}$ & $\begin{array}{r}\tau_{\mathrm{dc}} \\
(\mathrm{ps})\end{array}$ & \multicolumn{1}{c}{$\begin{array}{c}\tau_{\mathrm{ac}} \\
(\mathrm{ps})\end{array}$} \\
\hline $\mathrm{A}$ & 1.6 & 5.5 & 200 & 140 \\
$\mathrm{~B}$ & 1.25 & 1.8 & 70 & 40 \\
$\mathrm{C}$ & 1.5 & 0.6 & 25 & $\leqslant 25$ \\
$\mathrm{D}$ & 2.0 & 15.6 & 590 & $\geqslant 200$ \\
\hline \hline
\end{tabular}

well as the qualitative similarity to the temperature dependence of the mobility measured by dc transport on separate samples from the same wafer, is further verification of Eqs. (1) and (2).

While quantitative measurements of both the dc and ac conductivity of the same sample has proven difficult, since attaching dc leads significantly perturbs the rf current distribution, we can compare the ac and dc measurements on separate samples from the same wafer. We carried out this procedure on 12 samples from 4 different wafers, and summarize our results in the Table I. The microwave impedance displayed $R L C$ behavior for all samples measured. The transport time determined from dc measurements of the mobility is in reasonable agreement with the transport time determined from the frequency at which $\operatorname{Re}\left(Z_{2 \mathrm{DEG}}\right)=\operatorname{Im}\left(Z_{2 \mathrm{DEG}}\right)$ on separate samples from the same wafer. The data in Table I for all samples except the one from wafer $\mathrm{D}$ were measured in the dark, but we also find results consistent with dc measurements after illumination with a red light emitting diode (LED). For sample D, illumination was necessary to achieve the mobility quoted in Table I. ${ }^{5}$ However, due to the geometry for that sample, the unilluminated regions under the gate contributed significantly to the total circuit impedance, allowing only a lower limit on $\tau_{\text {tr }}$ to be determined.

We now turn to a more detailed study of the capacitive contacts. While we modeled the gate as a single capacitor above, a more complete model of the system would treat the capacitance and kinetic inductance of the system as distributed elements. The voltages and currents in such a system, pictured schematically in the inset of Fig. 2, obey a wave equation, with the wave velocity $\mathrm{v}$ given by $1 / \sqrt{\mathcal{L}_{K} \mathcal{C}}$ $=\sqrt{n e^{2} d / m^{*} \epsilon}$. [Here $\epsilon$ is the dielectric constant, $\mathcal{L}_{K}$ the (kinetic) inductance per unit length, and $\mathcal{C}$ the geometric capacitance per unit length.] $v$ is roughly 100 times slower than the speed of light in vacuum for typical parameters. In contrast, more conventional waveguides are dominated by the magnetic inductance with a wave velocity given by $1 / \sqrt{\mathcal{L}_{m} \mathcal{C}}$, with $\mathcal{L}_{m}$ the (magnetic) inductance per unit length; this is the speed of light in the medium. We note that this is a circuit description of the long wavelength limit of a $2 d$ plasmon, which has a dispersion curve given by ${ }^{6} \omega=\sqrt{k n e^{2} / 2 \epsilon m^{*}}$, in the absence of a highly conducting gate, and

$$
\omega^{2}=\frac{n e^{2}}{\epsilon m^{*}} k \frac{1}{1+\operatorname{coth}(k d)}
$$

in the presence of a highly conducting gate..$^{7-11}$

We now consider the full circuit diagram of our system. The circuit consists of two capacitive contacts in series with a section of ungated 2DEG. The impedance of the capacitive AIP license or copyright, see http://apl.aip.org/apl/copyright.jsp 
contact from the gate to the 2DEG at the point under the edge of the gate can be calculated exactly using transmission line theory. One finds

$$
Z_{\text {gate-2DEG }}=\sqrt{\frac{\mathcal{R}+i \omega \mathcal{L}_{K}}{i \omega \mathcal{C}}} \operatorname{coth}\left[L_{\text {gate }} \sqrt{i \omega \mathcal{C}\left(\mathcal{R}+i \omega \mathcal{L}_{K}\right)}\right]
$$

where $L_{\text {gate }}$ is the length of the gate, and $\mathcal{L}, \mathcal{R}$, and $\mathcal{C}$ are the (kinetic) inductance, resistance, and capacitance per unit length respectively. At high frequencies $\left(\omega \tau_{\mathrm{tr}} \gg 1\right)$, the real and imaginary parts are periodic in $k L_{\text {gate }}=(\omega / \mathrm{V}) L_{\text {gate }}$. On the other hand, the low frequency limit of Eq. (4) is

$$
\lim _{\omega \rightarrow 0}\left(Z_{\text {gate-2DEG }}\right) \approx \frac{1}{i \omega C}+\frac{1}{3}\left(R+i \omega L_{K}\right),
$$

where $R, L_{K}$, and $C$ are the total resistance, (kinetic) inductance, and capacitance, respectively, of the gated 2DEG. Thus, the dc limit is mainly a capacitor (as expected) plus some inductance and resistance.

We measure the equivalent of Eq. (4) (plus a small contribution from an ungated region) by connecting a circuit, shown in Fig. 2, for a resonant structure from wafer A with a gate length of $360 \mu \mathrm{m}$, width of $100 \mu \mathrm{m}$, distance from the 2DEG to gate $d$ of $5800 \AA$, and a gap between gates of only $60 \mu \mathrm{m}$. By reducing the length of the ungated region to $60 \mu \mathrm{m}$, we ensure that the gate to 2DEG contact impedance is a much larger contribution to the total circuit impedance than the ungated region, in contrast to the experiments described in the first half of this letter where the ungated region dominated the total circuit impedance.

The results are plotted in Fig. 2 for $T=0.3 \mathrm{~K}$, together with the prediction of Eq. (4). Since the density is determined independently and the geometry and mobility are known, there are no free parameters in the theory curve. The data agree well with the theory. (We used the measured audio frequency capacitance to determine the capacitance per unit length since the gate-to-2DEG separation as well as the dielectric constant of AlGaAs is uncertain at the 10\% level.) The slight rise in the value of the imaginary impedance with frequency is due to the small inductance of the ungated 2DEG.

In another sample from the same wafer (A) of the same resonant geometry but with dc leads, we measured the wave velocity versus density by applying a dc bias to the gates to vary the density. The wave velocity was determined from the frequency of the first peak in $\operatorname{Re}(Z)$ and the gate length via the relationship $v=2 L_{\text {gate }} f_{\text {peak }}$, which can be derived from Eq. 4. The result is shown in Fig. 3. The theory curve is generated from fundamental constants, $m^{*}=0.067 m$, and the value of $d / \epsilon$ determined from the audio frequency capacitance. The experimental error is due to an uncertainty of

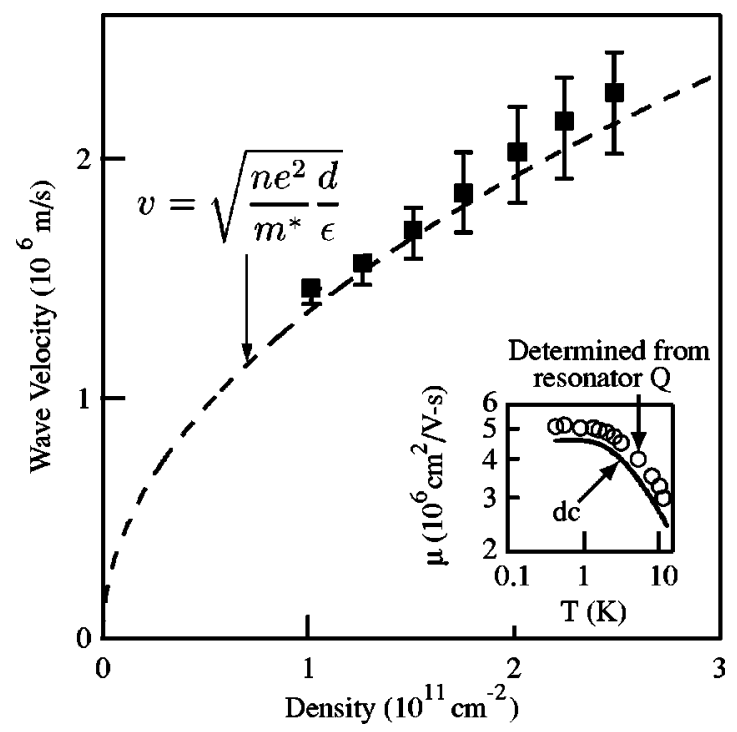

FIG. 3. Wave velocity vs density and temperature-dependent damping.

about $1 \mathrm{~mm}$ in the electrical length of the coax used to correct the microwave phase. The data agree with the model within the experimental uncertainties. We also measure the temperature dependence of the damping, and perform a oneparameter fit of $\operatorname{Re}(Z)$ to Eq. (4), varying only the mobility. The result is shown in the inset of Fig. 3, together with the dc mobility measured on the same sample. The two methods are in good agreement, further verifying our model.

The authors thank M. Roukes for loaning them the network analyzer necessary for these measurements and S. J. Allen, S. Das Sarma, A. Pinzcuk, and J. Zmuidzinas for useful discussions. One of the authors (P.J.B.) was supported in part by the Sherman Fairchild Foundation.

${ }^{1}$ Y. Yang, F. Agahi, D. Dai, C. Musante, W. Grammer, K. Lau, and K. Yngvession, IRE Trans. Microwave Theory Tech. 41, 581 (1993).

${ }^{2}$ S. Allen, D. Tsui, and F. DeRosa, Phys. Rev. Lett. 35, 1359 (1975).

${ }^{3}$ S. Allen, D. Tsui, and R. Logan, Phys. Rev. Lett. 38, 980 (1977).

${ }^{4}$ K. Hirakawa, K. Yamanaka, M. Grayson, and D. Tsui, Appl. Phys. Lett. 67, 2326 (1995)

${ }^{5} \mathrm{An}$ interesting topic for future research would be seeking to understand how the high frequency conductivity is modified in mesoscopic samples. See, for example, M. Büttiker and T. Christen, in Mesoscopic Electron Transport, edited by L. L. Sohn, L. P. Kouwenhoven, and G. Schön (Kluwer Academic, Dordrecht, The Netherlands, 1997), pp. 259-590. Since the width of our sample D is less than the calculated mean free path of $110 \mu \mathrm{m}$, such effects may already be significant.

${ }^{6}$ F. Stern, Phys. Rev. Lett. 18, 546 (1967).

${ }^{7}$ A. Eguiluz, T. Lee, J. Quinn, and K. Chiu, Phys. Rev. B 11, 4989 (1975).

${ }^{8}$ S. Das Sarma and A. Madhukar, Phys. Rev. B 23, 805 (1981).

${ }^{9}$ G. E. Santoro and G. F. Giuliani, Phys. Rev. B 37, 937 (1988).

${ }^{10}$ S. Das Sarma and E. H. Hwang, Phys. Rev. Lett. 81, 4216 (1998).

${ }^{11}$ D. Kainth, D. Richards, H. Hughes, M. Simmons, and D. Ritchie, Phys. Rev. B 57, 2065 (1998). 